\title{
Large-scale polarization of the microwave background and foreground
}

\author{
Angélica de Oliveira-Costa, ${ }^{1, *}$ Max Tegmark, ${ }^{1}$ Christopher O’Dell, ${ }^{2}$ Brian Keating, ${ }^{3}$ Peter Timbie, ${ }^{4}$ George Efstathiou, ${ }^{5}$ \\ and George Smoot ${ }^{6}$ \\ ${ }^{1}$ Department of Physics \& Astronomy, University of Pennsylvania, Philadelphia, Pennsylvania 19104, USA \\ ${ }^{2}$ Department of Astronomy, University of Massachusetts, Amherst, Massachusetts 01003, USA \\ ${ }^{3}$ Department of Physics, California Institute of Technology, Pasadena, California 91125, USA \\ ${ }^{4}$ Department of Physics, University of Wisconsin, Madison, Wisconsin 53706-1390, USA \\ ${ }^{5}$ Institute of Astronomy, University of Cambridge, Cambridge CB3 OHA, United Kingdom \\ ${ }^{6}$ Department of Physics, University of California, Berkeley, California 94720, USA
}

(Received 23 December 2002; published 29 October 2003)

\begin{abstract}
The DASI discovery of cosmic microwave background (CMB) polarization has opened a new chapter in cosmology. Most of the useful information about inflationary gravitational waves and reionization is on large angular scales where galactic foreground contamination is the worst, so a key challenge is to model, quantify, and remove polarized foregrounds. We use the POLAR experiment, COBE/DMR and radio surveys to provide the strongest limits to date on the $T E$ cross-power spectrum of the $\mathrm{CMB}$ on large angular scales and to quantify the polarized synchrotron radiation, which is likely to be the most challenging polarized contaminant for the WMAP satellite. We find that the synchrotron $E$ and $B$ contributions are equal to within $10 \%$ from $408-820$ $\mathrm{MHz}$ with a hint of $E$ domination at higher frequencies. We quantify Faraday rotation and depolarization effects in the two-dimensional $(\ell, \nu)$ plane and show that they cause the synchrotron polarization percentage to drop both towards lower frequencies and towards lower multipoles.
\end{abstract}

DOI: 10.1103/PhysRevD.68.083003

PACS number(s): 98.70.Vc, 98.65.Dx, 98.80.Es

\section{INTRODUCTION}

The recent discovery of cosmic microwave background (CMB) polarization by the DASI experiment [1] has opened a new chapter in cosmology-see Fig. 1. Although CMB polarization on degree scales and below can sharpen cosmological constraints and provide important cross-checks [2,3], the potential for the most dramatic improvements lies on the largest angular scales where it provides a unique probe of the reionization epoch and primordial gravitational waves. For instance, forecasts $[4,5]$ indicate that the Wilkinson Microwave Anisotropy Probe (WMAP) satellite can measure the reionization optical depth $\tau$ seventeen times more accurately using polarization information, and that polarization increases the sensitivity of the Planck satellite to tensor modes by a factor of 25 .

Unfortunately, these large scales are also the ones where polarized foreground contamination is likely to be most severe, both because of the red power spectra of the diffuse galactic synchrotron and dust emission and because they require using a large fraction of the sky, including less clean patches. The key challenge in the CMB polarization endeavor will therefore be modeling, quantifying and removing large-scale polarized galactic foregrounds. This is the topic of the present paper. We will use the POLAR experiment to provide the strongest limits to date on the cross-polarized microwave background and foreground fluctuations on large angular scales, and employ polarization sensitive radio surveys to further quantify the polarized synchrotron radiation, which is likely to be the most challenging contaminant in the polarization maps expected from the WMAP satellite.

\footnotetext{
*Email address: angelica@higgs.hep.upenn.edu
}

At microwave frequencies, three physical mechanisms are known to cause foreground contamination: synchrotron, freefree and dust emission. When coming from extragalactic objects, this radiation is usually referred to as point source contamination and affects mainly small angular scales. When coming from the Milky Way, this diffuse galactic emission fluctuates mainly on the large angular scales that are the focus of this paper. Except for free-free emission, all the above mechanisms are known to emit polarized radiation. In the near term, the best measurement of large-scale polarization will probably come from the WMAP satellite. At WMAP's frequency range $(22-90 \mathrm{GHz})$, synchrotron radiation is likely to be the dominant polarized foreground [4]. Unfortunately, we still know basically nothing about the polarized contribution of the galactic synchrotron component at CMB frequencies [4,24-29], since it has only been measured at lower frequencies and extrapolation is complicated by Faraday rotation. This is in stark contrast to the CMB itself, where the expected polarized power spectra and their dependence on cosmological parameters has been computed from first principles to high accuracy [30-33].

Polarization of the galactic continuum emission was first clearly detected in 1962 [34]. In the succeeding years, polarization measurements of the northern sky were made at frequencies between 240 and $1415 \mathrm{MHz}$ (see [35] and references therein) with resolutions of only a few degrees. No large-area survey has been published since the compendium of Brouw and Spoelstra [36] and high-resolution surveys have only begun to be made recently. The first major investigation done after [36] is that of [37], who observed a section of the galactic plane defined by $49^{\circ} \leqslant \ell \leqslant 76^{\circ}$ and $|b|$ $\leqslant 15^{\circ}$, at a frequency of $2.7 \mathrm{GHz}$. The study of [38] provides the highest resolution insight into the small-scale structure of the galaxy; however, this only covered a few areas of the sky 


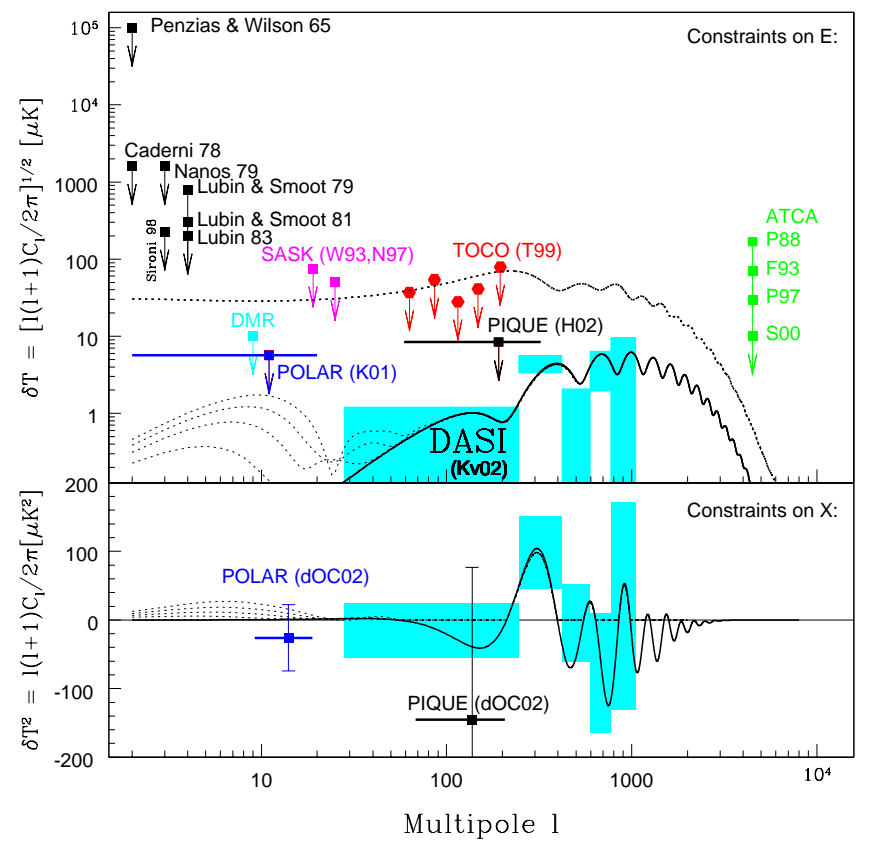

FIG. 1. Summary of constraints on polarization so far. From top to bottom, the three curves show the concordance model predictions for $C_{\ell}^{T}, C_{\ell}^{E}$ and $C_{\ell}^{X}$, respectively. Four reionization models with $\tau=0.1,0.2,0.3$ and 0.4 are also plotted (left dotted lines from bottom to top in both plots). The limits for $E$ are shown in the upper panel: Penzias and Wilson 65 [6], Caderni 78 [7], Nanos 79 [8], Lubin and Smoot 79 [9], Lubin and Smoot 81 [10], Sironi 98 [11], Lubin 83 [12], SASK (W93 [13],N97 [14]), TOCO (T99 hexagons [15]), P88 [16], F93 [17], P97 [18], S00 [19], DMR [20], PIQUE (H02 [21]) and POLAR (K01 [22]). The limits for $X$ are shown in the lower panel: PIQUE (d0C02 [23]) and POLAR (this work). The shaded regions are the DASI results (Kv02 [1]).

which were not larger than a degree or so across. Recently, two fully sampled polarimetric surveys were done at 2.4 $\mathrm{GHz}[39,40]$ and $1.4 \mathrm{GHz}[41,42]$. All of these highresolution surveys covered only regions near the galactic plane, so in order to use them for inferences relevant to CMB experiments, they need to be extrapolated both in galactic latitude and in frequency.

The rest of this paper is organized as follows. In Sec. II we review the basics of $\mathrm{CMB}$ and synchrotron polarization as well as our methods for measuring and modeling it. We present our results in Sec. III and discuss our conclusions in Sec. IV.

\section{PHENOMENOLOGY}

\section{A. Notation}

CMB measurements can be decomposed into three maps ( $T, E, B)$, where $T$ denotes the unpolarized and $(E, B)$ denote the polarized components, respectively. Note that an experiment that is insensitive to polarization does not measure $T$ but rather that total (unpolarized plus polarized) intensity; although this distinction has traditionally been neglected for CMB experiments where the polarization fraction is small, it is important both for foregrounds (which can be highly po- larized) and for precision $\mathrm{CMB}$ experiments. From these three maps we can measure a total of six angular power spectra, here denoted by $C_{\ell}^{T}, C_{\ell}^{E}, C_{\ell}^{B}, C_{\ell}^{X}, C_{\ell}^{Y}$ and $C_{\ell}^{Z}$, corresponding to the $T T, E E, B B, T E, T B$ and $E B$ correlations, ${ }^{1}$ respectively.

By parity, $C_{\ell}^{Y}=C_{\ell}^{Z}=0$ for scalar CMB fluctuations, but it is nonetheless worthwhile to measure these power spectra as probes of both exotic physics [44-46] and foreground contamination. $C_{\ell}^{B}=0$ for scalar CMB fluctuations to first order in perturbation theory [30-33]—-secondary effects such as gravitational lensing can create $B$ polarization even if there are only density perturbations present [47]. In the absence of reionization, $C_{\ell}^{E}$ is typically a couple of orders of magnitude below $C_{\ell}^{T}$ on small scales and approaches zero on the very largest scales.

The cross-power spectrum $C_{\ell}^{X}$ is not well suited for the usual logarithmic power spectrum plot, since it is negative for about half of all $\ell$ values [23]. A theoretically more convenient quantity is the dimensionless correlation coefficient

$$
r_{\ell}^{X} \equiv \frac{C_{\ell}^{X}}{\left(C_{\ell}^{T} C_{\ell}^{E}\right)^{1 / 2}}
$$

plotted on a linear scale in Fig. 2 (lower panel), since the Schwarz inequality restricts it to lie in the range $-1 \leqslant r_{\ell}^{X}$ $\leqslant 1 .^{2}$ From here on we use $r_{\ell}$ as shorthand for $r_{\ell}^{X}$. For more details about $r_{\ell}$ and how it depends on cosmological parameters, see Sec. II.b in [23].

\section{B. Our knowledge of synchrotron emission}

The galactic interStellar medium (ISM) is a highly complex medium with many different constituents interacting through a multitude of physical processes. Free electrons spiraling around the galactic magnetic field lines emit synchrotron radiation [48], which can be up to $70 \%$ linearly polarized (see $[49,50]$ for a review).

The power spectrum $C_{\ell}$ of synchrotron radiation is normally modeled as a power law in both multipole $\ell$ and frequency $\nu$, which we will parametrize as

$$
\delta T_{\ell}^{2}(\nu)=A\left(\frac{\ell}{50}\right)^{\beta+2} \text { with } A \propto \nu^{2 \alpha}
$$

where $\delta T_{\ell} \equiv\left[\ell(\ell+1) C_{\ell} / 2 \pi\right]^{1 / 2}$. This definition implies that $C_{\ell} \propto \ell^{\beta}$ for $\ell \gg 1$ and that the fluctuation amplitude $\propto \nu^{\alpha}$. The

\footnotetext{
${ }^{1}$ From here on, we adopt the notation $T T \equiv T, E E \equiv E, B B$ $\equiv B, T E \equiv X, T B \equiv Y$ and $E B \equiv Z$.

${ }^{2}$ Note that for experiments where CMB polarization is measured with a very low signal-to-noise ratio, $C_{\ell}^{X}$ is a more useful quantity than $r_{\ell}^{X}$. This is because they may be able to place upper and lower limits on $C_{\ell}^{X}$ but can place no meaningful limits on $r_{\ell}^{X}$ unless they can statistically rule out that $C_{\ell}^{E}$ in the denominator of Eq. (1).
} 


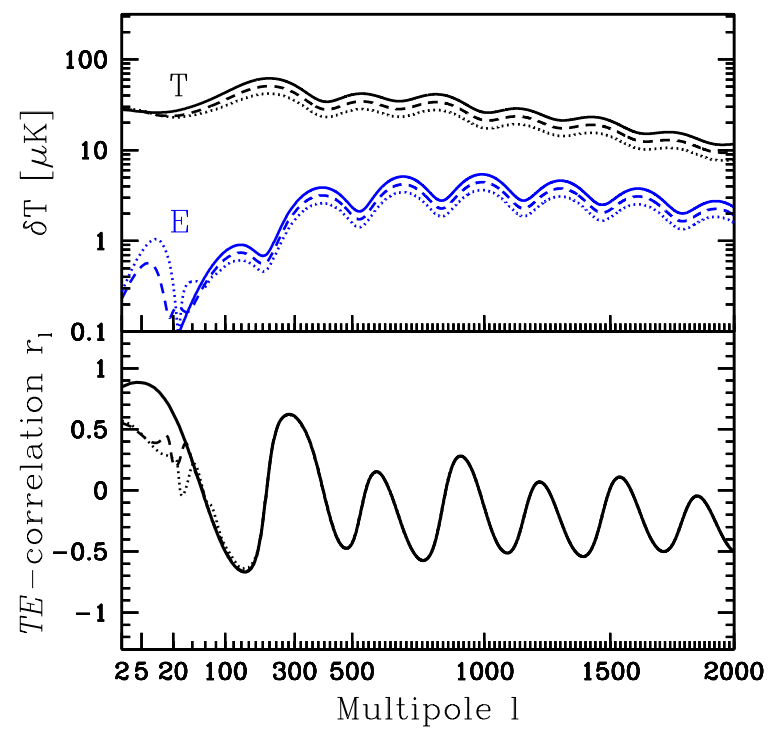

FIG. 2. Examples of CMB polarization, showing how the reionization optical depth $\tau$ affects the $T$ and $E$ power spectra (top) and the $T E$ correlation $r_{\ell}$ (bottom). Solid, dashed and dotted curves correspond to $\tau=0,0.2$ and 0.4 , respectively. As discussed in [23], changing the cosmological parameters affects the polarized and unpolarized power spectra rather similarly except for the cases of reionization and gravitational waves. In the reionization case, a new series of peaks are generated at large scales. Top panel: Although there is no visible change in $T$ at large scales, there is clearly a visible change in $E$ since the Sachs-Wolfe nuisance is unpolarized and absent. Lower panel: On small scales, reionization leaves the correlation $r_{\ell}$ unchanged since $C_{\ell}^{T}$ and $C_{\ell}^{E}$ are merely rescaled. On very large scales, $r_{\ell}$ drops since the new polarized signal is uncorrelated with the old unpolarized Sachs-Wolfe signal. On intermediate scales $\ell \geq 20$, oscillatory correlation behavior is revealed for the new peaks. For more details about CMB polarization and reionization see [43].

standard assumption is that the total intensity has $\alpha \approx-2.8$ with variations of order 0.15 across the $\mathrm{sky}^{3}[51]$.

As to the power spectrum slope $\beta$, the $408 \mathrm{MHz}$ Haslam map $[55,56]$ suggests $\beta$ of order -2.5 to -3.0 down to its resolution limit of $\sim 1^{\circ 4}[58-61]$. A similar analysis done on the $2.3 \mathrm{GHz}$ Rhodes map of resolution 20' [53] gives $\beta$ $=-2.92 \pm 0.07$ [62] (flattening to $\beta \approx-2.4$ at low galactic latitudes [29]).

For the polarized synchrotron component, our observational knowledge is, unfortunately, not as complete. To date, there are measurements of the polarized synchrotron power spectrum obtained basically from three different surveys

\footnotetext{
${ }^{3}$ Because the spectral index $\alpha$ depends on the energy distribution of relativistic electrons [48], it may vary somewhat across the sky. One also expects a spectral steepening towards higher frequencies, corresponding to a softer electron spectrum ([52]; Fig 5.3 in [53]). A recent analysis done at $22 \mathrm{MHz}$ [54] shows that $\alpha$ varies slightly over a large frequency range.

${ }^{4}$ Although the interpretation is complicated by striping problems [57].
}
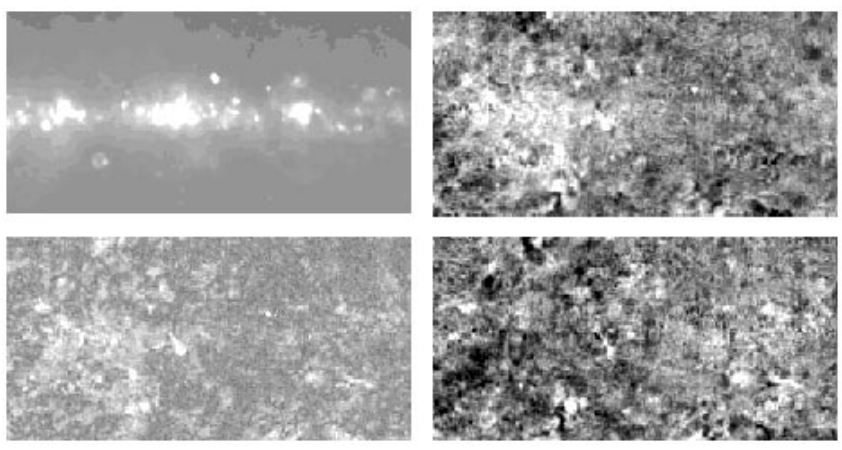

FIG. 3. The nature of the galactic synchrotron emission. Clockwise from top left, the panels show Stokes $T, U, Q$, and $P$ (defined as $P=\sqrt{Q^{2}+U^{2}}$ ) from Block 3 of the Parkes $2.4 \mathrm{GHz}$ Survey of the Southern Galactic Plane.

[63]: the Leiden surveys ${ }^{5}$ [36,35], the Parkes $2.4 \mathrm{GHz}$ Survey of the Southern Galactic Plane ${ }^{6}[39,40]$, and the Medium Galactic Latitude Survey ${ }^{7}[41,42,64]$.

These measurements exhibit a much bluer power spectrum in polarization than in intensity, with $\beta$ in the range from 1.4 to $1.8[4,24-29]$. These results are usually taken with a grain of salt when it comes to their implications for CMB foreground contamination, for three reasons:

(1) Extrapolations are done from low to high galactic latitudes.

(2) Extrapolations are done from low to high frequencies.

(3) Much of the available data is undersampled.

The Leiden surveys extend to high galactic latitudes and up to $1.4 \mathrm{GHz}$ but are unfortunately undersampled, while the Parkes and the Medium galactic Latitude Surveys only probe regions around the galactic plane. In the following three sections, we will discuss these three problems in turn.

\section{The latitude extrapolation problem}

Although only high galactic latitudes are relevant for CMB work, most of the data used for understanding the polarized CMB foreground contamination are at low galactic latitudes. Figure 3 shows that whereas the total intensity of the synchrotron emission depends strongly on the galactic latitude, the polarized component is approximately independent of galactic latitude-indeed, in the three polarized im-

\footnotetext{
${ }^{5}$ The observations done by Brouw and Spoelstra covered almost $40 \%$ of the sky extending to high galactic latitudes. Using the same instrument, they observed the polarized galaxy in $Q$ and $U$ in five frequencies from $408 \mathrm{MHz}$ up to $1.4 \mathrm{GHz}$ and with angular resolutions from $2.3^{\circ}$ at $408 \mathrm{MHz}$ up to $0.6^{\circ}$ at $1.4 \mathrm{GHz}$. Unfortunately this data was also undersampled, making it difficult to draw inferences about its polarized power spectrum.

${ }^{6}$ This survey covers a strip $127^{\circ}$ long and at least $10^{\circ}$ wide centered in the galactic plane, with a resolution of FWHM $=10.4^{\prime}$. It is publically available at $h t t p: / / w w w . u q . e d u . a u / \sim$ roy/

${ }^{7}$ The Medium Galactic Latitude Survey maps the galactic plane within $\pm 20^{\circ}$, with a resolution of $\mathrm{FWHM}=9.35^{\prime}$ at $2.4 \mathrm{GHz}$. This survey is partially available at http://www.mpifr-bonn.mpg.de/staff/ buyaniker/index.htm
} 


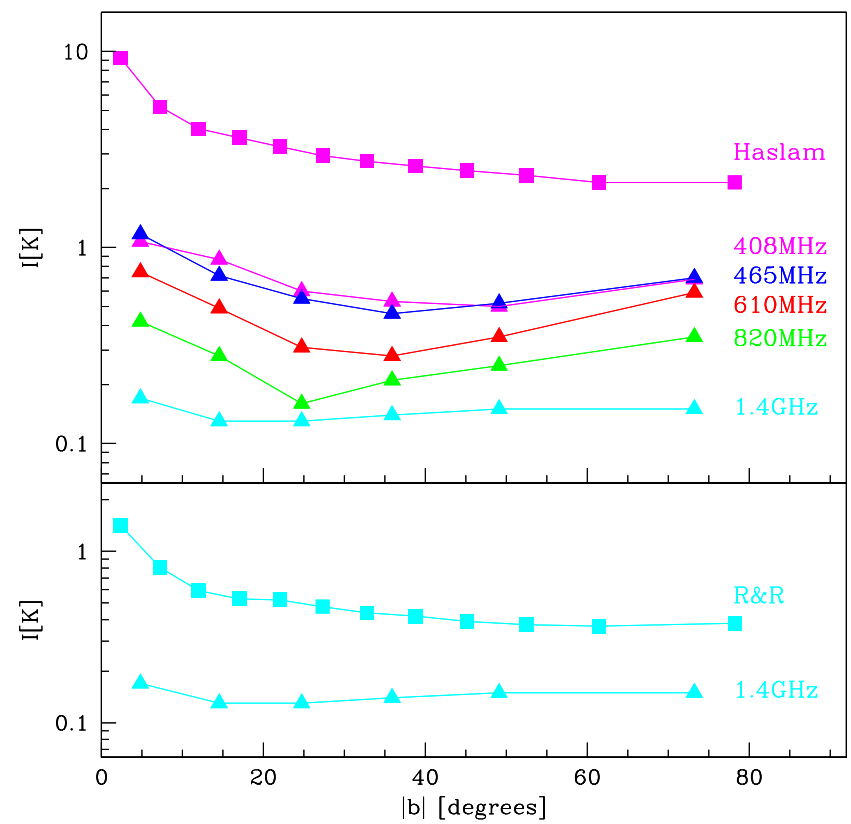

FIG. 4. The polarized and total synchrotron component as a function of the galactic latitude. Each of five Leiden polarized surveys was divided in six slices of equal area, we then calculated the mean intensity (defined as $P=\sqrt{Q^{2}+U^{2}}$ ) for each of those slices. A similar procedure was used for the polarization insensitive Haslam and Reich and Reich surveys, but 12 slices were chosen instead. The top panel show the results from the five Leiden surveys plus the $408 \mathrm{MHz}$ Haslam data, while the bottom panel show the results from the Leiden $1.4 \mathrm{GHz}$ survey and $1.42 \mathrm{GHz}$ Reich and Reich data. Comparison between polarized components and the total intensity at the same frequency illustrates that the polarized synchrotron is almost independent of the galactic latitude while the unpolarized emission is strongly concentrated in the galactic plane.

ages, it is difficult to distinguish the galactic plane at all. As noticed long ago by [40], there is a faint, quasiuniform polarized component of the galactic polarized emission in their survey, upon which the emission from other features is superimposed: towards the higher latitudes, this faint component appears similar in both structure and intensity to the correspondent lower latitude emission. This well-known empirical result can be also seen (in a more quantitative way) in the Leiden surveys. Figure 4 shows that in the frequency range between $408 \mathrm{MHz}$ to $1.4 \mathrm{GHz}$, the polarization intensity $P\left(P=\sqrt{Q^{2}+U^{2}}\right)$ is basically constant as the galactic latitude $|b|$ increases, whereas the polarization insensitive surveys (such as the $408 \mathrm{MHz}$ Haslam and the $1420 \mathrm{MHz}$ Reich and Reich [65]) have the bulk of their emission coming from the galactic plane.

The usual interpretation of this very weak latitude dependence of polarized synchrotron radiation is that the signal is dominated by sources that are nearby compared to the scale height of the galactic disk, with more distant sources being washed out by depolarization (to which we return in the next section). As a result, having well-sampled polarized maps off the galactic plane at the same frequencies would not be expected to affect our results much, since they would be similar to those in the plane. This issue, however, deserves more work as far as extrapolation to CMB frequencies is concerned: the latitude dependence may well return at higher frequencies as depolarization becomes less important, thereby revealing structure from more distant parts of the galactic plane. In this case, extrapolating from an observing region around the galactic plane to higher latitudes may well result in less small-scale power in the angular distribution.

If we are lucky, many of the complications of extrapolating to higher latitude may largely cancel out the complications of extrapolating to higher frequency, thereby making it easier to quantify the polarized CMB foreground problem. The reason for optimism is the following: at high latitudes (which is all that really matters for CMB research), the foreground signal will be entirely due to nearby emission within the scale height of the thick galactic disk; and at low frequencies in the galactic plane (which is where we have really good data), the polarized signal we see may well be dominated by such nearby emission, with emission from more distant regions in the galactic disk hidden by depolarization.

\section{Faraday rotation, depolarization and the frequency extrapolation problem}

The plane of a polarized wave may be regarded as the sum of two circularly polarized components of opposite handedness. In an ionized medium with a non-zero magnetic field, these two components propagate with different phase velocities, which will result in a rotation of the plane of polarization of the linearly polarized radiation. This rotation, known as the Faraday rotation, ${ }^{8}$ produces a change in polarization angle $\Delta \theta$ of

$$
\Delta \theta=0.81 \lambda^{2} \int_{0}^{L} n_{e} B_{\|} d L=\lambda^{2} R M(\mathrm{rad})
$$

where $\lambda$ is the wavelength given in meters and the quantity $\Delta \theta / \lambda^{2}$ is called the rotation measure [(RM), usually expressed in units of $\left.\mathrm{rad} \mathrm{m}^{-2}\right]$. The integral is done over the line of sight from us to the emitting region at a distance $L$ in pc, $n_{e}$ is the free electron density in $\mathrm{cm}^{-3}$, and $B_{\|}$is the magnetic field parallel to the line of sight in $\mu \mathrm{G}$.

From Eq. (3) it is easy to see that observations of this synchrotron radiation in several frequencies allows the determination of rotation measures in the diffuse radiation. From the obtained structure in the rotation measure on different scales, we can obtain information on the magnetic field parallel to the line of sight, weighted with electron density - an example of this method can be found in [68]. In radio astronomy, Faraday rotation has become one of the main tools to investigate the interstellar magnetic field (see, e.g. $[69,70])$.

It is important to point out, however, that Faraday rotation can only change the polarization angle and not the polarized intensity $P$. The fact that we do see structure in $P$ that is not correlated with a counterpart in intensity $T$ implies that part

\footnotetext{
${ }^{8} \mathrm{~A}$ detailed discussion of the Faraday rotation and depolarization effects as well as their importance in astrophysical observations is in given in [67].
} 
of the radiation has been depolarized [38]. A simple visual comparison of the total intensity and polarized maps of the same region in the sky of the Parkes $2.4 \mathrm{GHz}$ survey shows depolarization at work (see Fig. 3): many sources which present an intense total emission do not show a counterpart in the polarized maps; similarly bright regions of extended polarization are not connected with unpolarized sources. A more detailed study of this same survey reached similar conclusions: Giardino et al. [29] showed that the $E$ and $B$ power spectra were dominated by changes in the polarization angle rather than by changes in the polarized intensity, suggesting that Faraday rotation was playing a significant role. ${ }^{9}$

Depending on the frequency and beamwidth used, depolarization can play an important role in polarization studies of the galactic radio emission [35]. As discussed by Cortiglioni and Spoelstra [71], depolarization can have four causes: (1) differential polarization along the line of sight, (2) differential polarization across the beam, (3) differential Faraday rotation across the beam, and (4) differential Faraday rotation and polarization across the bandwidth. If the bandwidth is very narrow, we can neglect item (4); also, if the polarized data have been sufficiently sampled, smoothing it to a largest beam may inform us about items (2) and (3), leaving us with item (1) as the expected main source of depolarization. ${ }^{10}$

Because of the complicated interplay of these mechanisms, we should expect both the amplitude and the shape of the polarized synchrotron power spectrum to change with frequency. We will therefore take an empirical approach below and use the available data to map out (for the first time) the two-dimensional region in the $(\ell, \nu)$ plane where Faraday rotation and depolarization are important.

\section{Incomplete sky coverage and the undersampling problem}

For the case of undersampling in the Leiden surveys, some authors have overcome this problem by doing their Fourier analysis over selected patches in the sky where they believe the average grid space in the patch is close to the

\footnotetext{
${ }^{9}$ Although at first glance the images in Fig. 3 suggest that the polarized and unpolarized components are uncorrelated, [40] found that for some patches in their images there is a good correlation between the polarized and total power intensities. Therefore they conclude that a good fraction of the polarized emission seen over the plane was caused by changes in synchrotron emissivity rather than any depolarization or Faraday rotation of the synchrotron background. According to [40], variations in synchrotron emission can be caused by increases in the density of relativistic electrons (due to SNRs), and/or variations in the magnetic field intensity. It is important to point out that the relative importance of these two mechanisms (Faraday rotation and depolarization and changes in the synchrotron emissivity of the source regions) over the galactic plane region are currently unknown [66].

${ }^{10}$ In the case of Leiden surveys, item (4) is negligible. Based on previous analysis done over the galactic loops at $1.4 \mathrm{GHz}[72,73]$, Spoelstra [35] argued that items (2) and (3) have a relatively minor contribution to the depolarization in those surveys. Leaving, therefore, differential polarization along the line of sight as the main source of depolarization.
}

map's beam size, so that they can apply a Gaussian smoothing on it-this is well explained and illustrated in [27]. Fortunately, we can eliminate this problem by measuring the power spectra with the matrix-based quadratic estimator technique that has recently been developed for analyzing CMB maps $[74,75,23]$.

Although the undersampling and partial sky coverage results in unavoidable mixing between different angular scales $\ell$ and polarization types ( $E$ and $B$ ), this mixing (also known as leakage) is fully quantified by the window functions that our method computes [75] and can therefore be included in the statistical analysis without approximations. Specifically, we compute the six power spectra $(T, E, B, X, Y, Z)$ described in Sec. II A so that the leakage, if any, is minimal.

In [75] it was argued that susceptibility to systematic errors could be reduced by choosing the "priors" that determine the quadratic estimator method to have vanishing cross-polarizations, $X=Y=Z=0$, and it was shown that this simplification came at the price of a very small (percent level) increase in error bars. In Appendix A of [23], it was shown that this choice has an important added benefit: exploiting a parity symmetry, it eliminates 14 out of the 15 leakages, with only the much discussed [75,32,76-79] $E$ $-B$ leakage remaining. In [80] it was shown that even the remaining $E-B$ leakage can, in principle, be removed. Unfortunately, this technique cannot be applied here, since it works only for a fully sampled two-dimensional map.

\section{RESULTS}

\section{A. POLAR power spectra}

POLAR was a ground-based CMB polarization experiment that operated near Madison, Wisconsin [22,81,82]. It used a simple drift-scan strategy, with a $7^{\circ}$ FWHM beam at 26-30 GHz, and simultaneously observed the Stokes parameters $Q$ and $U$ in a ring of declination $\delta=43^{\circ}$. Because POLAR was insensitive to the unpolarized CMB component, we cross-correlate their $Q$ and $U$ data with the $T$-data from the COBE/DMR map [83].

\section{Quadratic estimator analysis}

We measure the six power spectra described in Sec. II A using the quadratic estimator method exactly as described in [75]. We computed fiducial power spectra with the CMBFAST software [84] using cosmological parameters from the concordance model from [85] (that of [86] is very similar). Table I shows the result of our band-power estimation. The values shown in parentheses in the rightmost column of this table are our 2- $\sigma$ upper limits. In these calculations, we used five multipole bands of width $\Delta \ell=6$ for each of the six polarization types $(T, E, B, X, Y, Z)$, thereby going out to $\ell=30$, and we average the measurements together with inverse-variance weighting into a single number for each polarization type to minimize noise.

We used the combined DMR $53+90 \mathrm{GHz}$ data to obtain good sensitivity to the unpolarized component. We perform our analysis using strips of the DMR data of width $\pm 15^{\circ}$ around the POLAR declination-we found that further in- 
TABLE I. POLAR-DMR power spectrum.

\begin{tabular}{lccc}
\hline \hline & $\ell_{\mathrm{eff}} \pm \Delta \ell$ & $\delta \mathrm{T}^{2} \pm \sigma\left(\mu \mathrm{K}^{2}\right)$ & $\delta \mathrm{T}(\mu \mathrm{K})^{\mathrm{a}}$ \\
\hline$T$ & $15.6 \pm 6.6$ & $487.0 \pm 270.6$ & $22.1_{-5.5}^{+7.4}$ \\
$E$ & $12.6 \pm 4.5$ & $-9.9 \pm 32.0$ & $<4.7(7.4)$ \\
$B$ & $12.6 \pm 4.5$ & $13.9 \pm 32.0$ & $<6.8(8.8)$ \\
$X$ & $14.0 \pm 4.8$ & $-26.0 \pm 48.5$ & $<8.7(11.1)$ \\
$Y$ & $14.0 \pm 4.8$ & $-0.1 \pm 48.5$ & $<7.0(9.8)$ \\
$Z$ & $11.4 \pm 2.9$ & $-50.0 \pm 31.6$ & $<6.6(10.7)$
\end{tabular}

$\overline{{ }^{a} \text { Values in parentheses are 2- } \sigma \text { upper limits. Cross-correlation upper }}$ limits refer to $|X|,|Y|$ and $|Z|$.

creasing in the width of these disks did not significantly tighten our constraints. Finally, we eliminated sensitivity to offsets by projecting out the mean (monopole) from the $T, Q$ and $U$ maps separately.

The detection of unpolarized power is seen to be consistent with that published by the DMR [83] group. Table I shows that we detect no polarization or cross-polarization of any type, obtaining mere upper limits, just as the models predict. The window functions reveal substantial leakage between $E$ and $B$, so that the limits effectively constrain the average of these two spectra rather than both separately. This large leakage is due to the one-dimensional nature of the POLAR dataset, and can be completely eliminated with a fully sampled two-dimensional map [80].

Finally, we perform the same analysis described above by replacing the DMR stripe with a similar stripe selected from the $408 \mathrm{MHz}$ Haslam map (which was smoothed to $7^{\circ}$ and scaled to $30 \mathrm{GHz}$ using $\beta_{T}=-3$ ). We detected no crosspolarization of any type between POLAR and the Haslam map, obtaining a mere upper limit of $|X| \lesssim 11.0 \mu \mathrm{K}$ (or a 2- $\sigma$ upper limit of $15.4 \mu \mathrm{K})$.

\section{Likelihood analysis}

We complement our band-power analysis with a likelihood analysis where we assumed that $B=0$. Specifically, we set $B=Y=Z=0$ and take each of the remaining power spectra $(T, E, X)$ to be constant out to $\ell=30$.

We first perform a simple one-dimensional likelihood analysis for the parameter $E$ using the POLAR data alone (discarding the DMR information), obtaining the likelihood function in excellent agreement with that published by [22] — see Figure 5 (right panel, thin line). A similar onedimensional likelihood analysis for the parameter $T$ using the DMR data alone produces $T \approx 28 \mu \mathrm{K}$, consistent with that of the DMR team [83] (left panel, thin line). We then compute the likelihood function including both POLAR and DMR data in the three-dimensional space spanned by $\left(T, E, r_{\ell}\right)$ and compute constraints on individual parameters or pairs by marginalizing as in [85]. Once again, we obtain a $T$-measurement in complete agreement with that for the DMR team (left panel, thicker line).

Figure 6 shows our constraints in the $\left(E, r_{\ell}\right)$ plane after marginalizing over $T$. It is seen that our constraints on the cross-polarization are weaker than the Schwarz inequality $\left|r_{\ell}\right| \leqslant 1$, so in this sense the data have taught us nothing new.

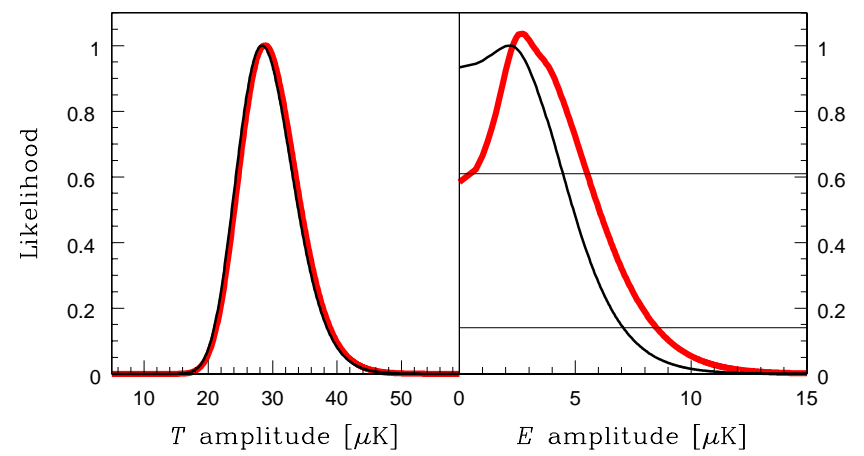

FIG. 5. Likelihood results using the $E$-polarized information alone (right panel, thin line), using $T$ information alone (left panel, thin line), and using both POLAR and DMR $T$ information and marginalizing (thicker lines). From top to bottom, the two horizontal lines correspond to $68 \%$ and $95 \%$ confidence limits, respectively.

The likelihood function is seen to be highly non-Gaussian, so obtaining statistically meaningful confidence limits (which is of course uninteresting in our case, since the constraints are so weak) would involve numerically integrating the likelihood function. Since $r_{\ell}$ is expected to oscillate between positive and negative values, using a flat (constant) $r_{\ell}$ in the likelihood analysis runs the risk of failing to detect a signal that is actually present in the data, canceling out positive and negative detections at different angular scales. This is not likely to have been a problem in our case, since $r_{\ell}$ is uniformly positive in our sensitivity range $\ell=14 \pm 5$ for the concordance model.

Figure 1 compares our results with all other polarization constraints published to date.

\section{B. The Leiden power spectra}

\section{Basic power spectra}

For the Leiden surveys, our analysis was performed using 10 multipole bands of width $\Delta \ell=10$ for each of the six

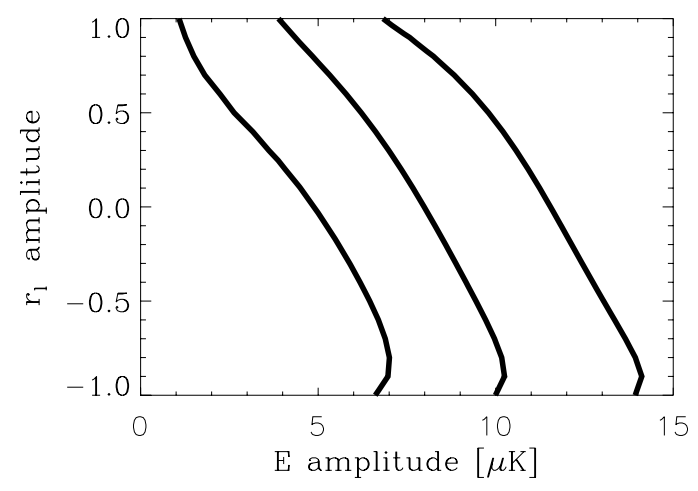

FIG. 6. Joint constraints on $E$ polarization and $r_{\ell}$ after marginalizing over $T$. From left to right, the contours show that the likelihood function has dropped to $e^{-1.1}, e^{-3.0}$ and $e^{-4.6}$ times its maximum value, which would correspond to $68 \%, 95 \%$ and $99 \%$ limits if the likelihood were Gaussian. For comparison, the concordance model predicts $\left(E, r_{\ell}\right)=(0.001,0.66)$ at $\ell=14$, the center of our window function for $X$ (see Table I). 

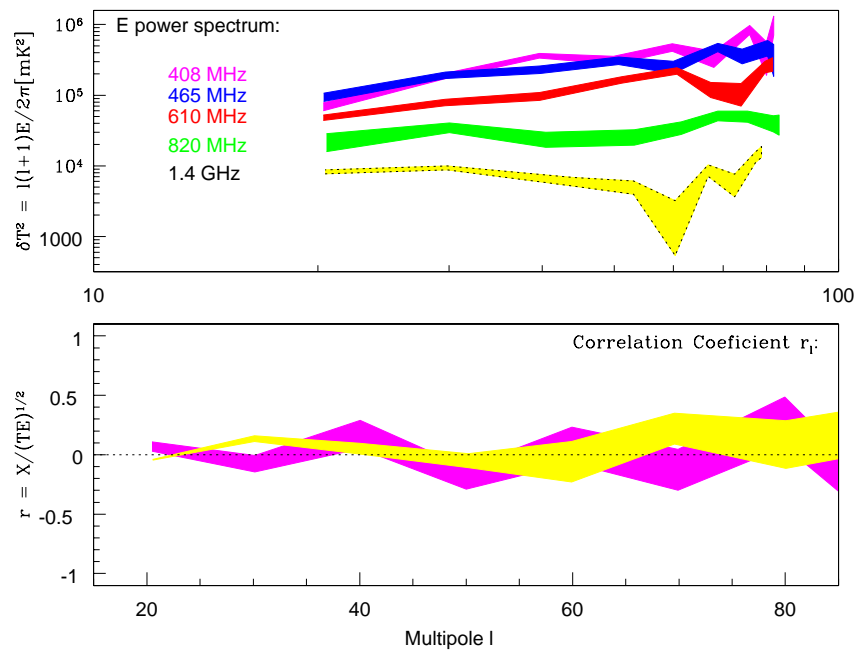

FIG. 7. Power spectra of the Leiden data. Top panel shows the $E$ power spectra for the five Leiden frequencies going from 408 to $1411 \mathrm{MHz}$, while the bottom panel shows the $X$ cross-power spectrum $r_{\ell}$ for two of the five Leiden frequencies (each frequency is represented by the same color/shade in both plots). Intrinsic EB correlation could be present but masked by Faraday rotation, since random rotations of the polarization angle would cause correlations to average to zero.

polarization types $(T, E, B, X, Y, Z)$, thereby going out to $\ell$ $=100$. We used the Haslam map for the unpolarized component $T$, scaled and smoothed to match Leiden's five different frequencies. A galactic cut of $|b|=25^{\circ}$ was applied in order to match the POLAR observing region. We iterated the QE method once and chose the second prior to be a simple power law model consistent with the original measurement for the $T, E$ and $B$ power. The priors for $X, Y$ and $Z$ were set as zero.

Figure 7 shows the $E$ power spectra (top) and $r_{\ell}$ correlation coefficient (bottom) of the Leiden surveys. We find that all power spectra are well approximated by powers laws as in Eq. (2). The best fit normalizations $A$ and slopes $\beta$ for $E$ and $B$ are shown in Table II. The values of $\beta$ are consistent with previous analyses [4,24-29], showing that the slopes get redder as frequency increases.

For all Leiden surveys, the $X$ and $Y$ power spectra are found to be consistent with zero-the $2.4 \mathrm{GHz}$ Parkes survey had a similar finding for $X$ [29]. These are not surprising results: if Faraday rotation makes the polarized and unpolarized components to be uncorrelated (see Fig. 3), it is natural

TABLE II. Normalization and spectral index. ${ }^{\mathrm{a}}$

\begin{tabular}{ccccc}
\hline \hline $\begin{array}{c}\nu \\
(\mathrm{GHz})\end{array}$ & $\begin{array}{c}A_{E} \\
\left(m K^{2}\right)\end{array}$ & $\beta_{E}$ & $\begin{array}{c}A_{B} \\
\left(m K^{2}\right)\end{array}$ & $\beta_{B}$ \\
\hline 0.408 & 5.5 & -0.5 & 5.7 & -0.4 \\
0.465 & 5.4 & -1.0 & 5.4 & -0.5 \\
0.610 & 5.1 & -1.0 & 5.1 & -0.8 \\
0.820 & 4.5 & -1.5 & 4.6 & -1.8 \\
1.411 & 3.9 & -1.9 & 3.6 & -2.6 \\
\hline \hline
\end{tabular}

$\overline{{ }^{a} \text { All fits are normalized at } \ell=50 \text {, i.e., } \delta \mathrm{T}_{\ell}^{2}=A(\ell / 50)^{\beta+2}}$. to expect that $X, Y=0$. However, at the $\mathrm{CMB}$ frequencies (where the effects of Faraday rotation and depolarization are unimportant) this should not be the case.

To study the frequency dependence, we average the 10 multipole bands of the Leiden power spectrum measurements together into a single band for each polarization type to reduce noise. From these results, we fit the average frequency dependence (for the $25^{\circ}$ cut data) as a power law as in Eq. (2) with slope $\alpha_{E}=-1.3$ and $\alpha_{B}=-1.5$ for $E$ and $B$ polarization, respectively.

\section{Is it $E$ or is it B?}

An interesting question about polarized foregrounds is how their fluctuations separate into $E$ and $B$. Although many authors initially assumed that foregrounds would naturally produce equal amounts of $E$ and $B$, Zaldarriaga [77] showed that this need not be the case. There are plausible scenarios where the foreground polarization direction could preferentially be aligned with or perpendicular to the gradient of polarized intensity, thereby producing more $E$ than $B$. In contrast, it is more difficult to contrive scenarios with more $B$ than $E$, since they require polarizations preferentially making a $45^{\circ}$ angle with the gradient.

Early studies $[25,29]$ have indicated that $E \approx B$ at $2.4 \mathrm{GHz}$ in the galactic plane. However, these analyses used Fourier transforms and spin-2 angular harmonic expansions, respectively, without explicitly computing the window functions quantifying the leakage between $E$ and $B$. This leakage is expected to be important both on the scale of the Parkes stripe thickness and on the pixel scale $[75,80]$, and would have the effect of mixing $E$ and $B$ power, reducing any $E / B$ differences that may actually be present. Moreover, no study of the $E / B$ ratio has ever been done on the large angular scales $(\ell \leqq 40)$, which are the most important for constraining reionization and inflationary gravitational waves.

We therefore perform a likelihood analysis of the Leiden surveys specifically focusing on this question, and we include an exact treatment of the leakage. The likelihood analysis of the data is done with two free parameters corresponding to the overall normalization of the $E$ and $B$ power spectra, and we assume that they both have the same power law shape given by the slopes $\beta_{E}$ from Table II. The results are shown in Fig. 8. Note that the $E$ and $B$ amplitudes are consistent with being equal to high accuracy at 408, 465, 610 and $820 \mathrm{MHz}$. At the highest frequency of $1.4 \mathrm{GHz}$, however, we see a hint of an $E$ excess at the $30 \%$ level, but this is only significant at a level of around $95 \%$. This hint is intriguing, since it can in principle be given a natural physical interpretation. It may be that synchrotron polarization has $E>B$ at $\mathrm{CMB}$ frequencies, and that Faraday rotation is hiding this underlying asymmetry at low frequencies. If the Faraday effect rotates each polarization angle by a random amount for all practical purposes, this will destroy any intrinsic alignment between the direction of the polarization and the direction of the local intensity gradient and therefore produce equal amounts of $E$ and $B$ signal. 

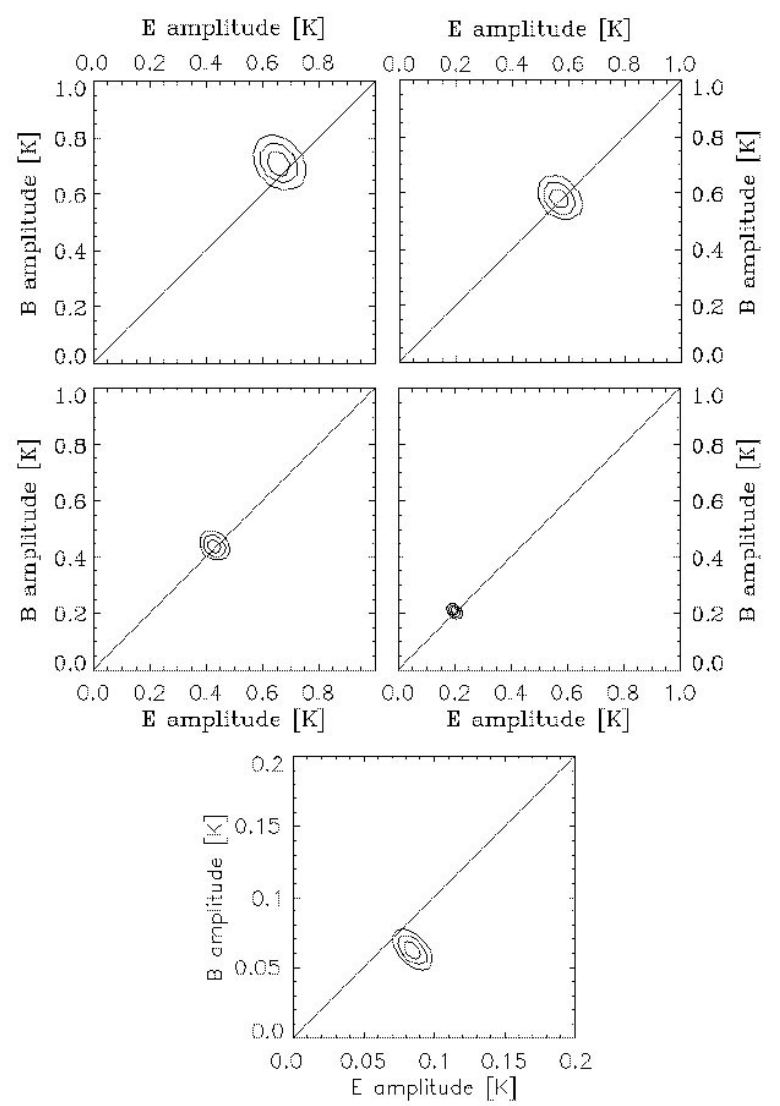

FIG. 8. $E$ and $B$ likelihood values for the Leiden surveys. From top to bottom, and from left to right, the likelihoods are for the frequencies 408, 465, 610, 820 and $1411 \mathrm{MHz}$. As the survey's frequency increases, the Faraday rotation reduces and we start to see a slight hint of an $E$ excess. For all likelihoods, the contours correspond to $68 \%, 95 \%$ and $99 \%$ limits. The diagonal lines correspond to $E=B$.

\section{Quantifying the importance of Faraday rotation and depolarization for the $\mathrm{CMB}$}

The key challenge for modeling synchrotron polarization as a CMB foreground is to answer the following question: above which frequency are the effects of Faraday rotation and depolarization so small that our measurements can be safely extrapolated up to CMB frequencies? From an analysis of the Leiden surveys, Spoelstra [35] found an upper limit for RM of $35 \mathrm{rad} \mathrm{m}^{-2}$. Setting $\Delta \theta=1 \mathrm{rad}$ in Eq. (3), this suggests that the Faraday rotation becomes irrelevant somewhere around $2 \mathrm{GHz}$. However, considering that the determination of RM is poor in many parts of these surveys, this $2 \mathrm{GHz}$ value is questionable. Moreover, because of the importance of depolarization which affects large scales more than small scales, we should expect the answer to depend on the angular scale $\ell$ considered.

Let us now quantify this empirically. Figure 9 shows the synchrotron power spectra as a function of frequency for a sample of angular scales $\ell$. Using the fits from Table II and Eq. (2) suggests that the polarization percentage $p$ $\equiv \delta T_{\ell}^{E} / \delta T_{\ell}^{T}$ saturates to a constant value for $\nu \gg 1 \mathrm{GHz}$ at $\ell=50, \nu \gg 4 \mathrm{GHz}$ at $\ell=14$ and $\nu \gg 10 \mathrm{GHz}$ at $\ell=2$. This

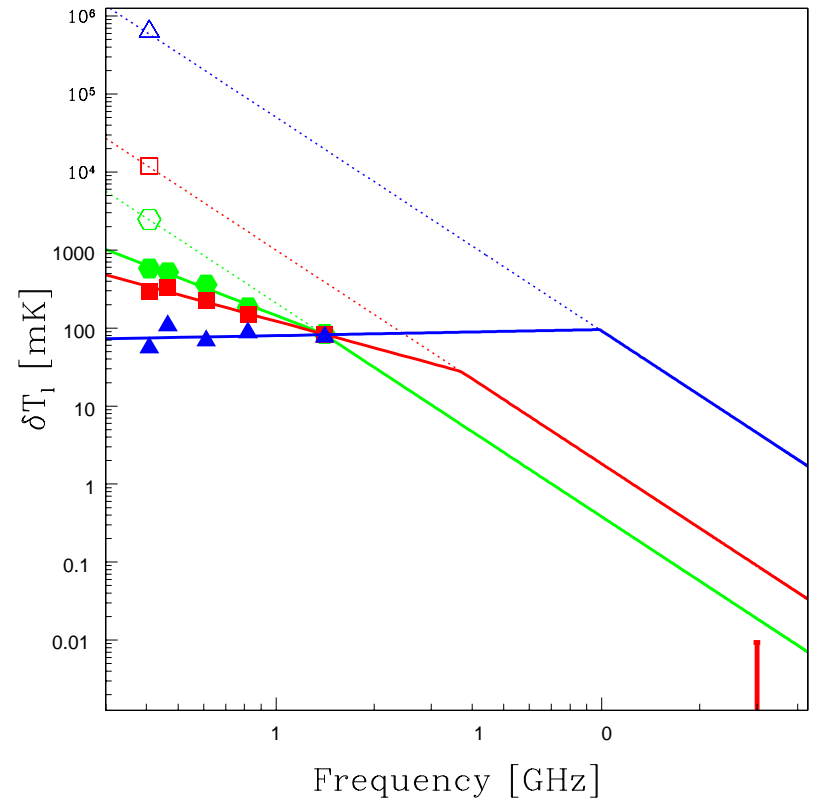

FIG. 9. The E-polarized (solid) and unpolarized (dashed) power spectra $\delta T_{\ell}$ of galactic synchrotron emission are plotted as a function of frequency for multipoles $\ell=2$ (triangles), $\ell=14$ (squares) and $\ell=50$ (hexagons) using the fits from Table II (corresponding to a $25^{\circ}$ galactic cut data). The $T$ curves (dashed line) assume $\alpha$ $=-2.8$. For comparison, the POLAR upper limit of $E<7.4 \mu \mathrm{K}$ centered in $\ell \sim 14$ (see Table I) is shown in the lower right corner. Comparing this with the squares implies either a low synchrotron polarization percentage or a steeper spectral index (lower $\alpha$ ).

suggest the following universal behavior. ${ }^{11}$ At high frequencies, where the Faraday rotation and depolarization effects are unimportant and the polarized fluctuations simply constitute some constant fraction of the total fluctuations, we can use the same $\alpha$ for polarized and total synchrotron radiation in the CMB range. However, moving to the left in Fig. 9, one reaches a critical frequency $\nu_{*}$ below which the Faraday rotation and depolarization effects suppress the polarized fluctuations. At this point, the power law changes asymptotes from a steeper (solid/dashed lines) to a shallower (just solid lines) power law, and the critical frequency $\nu_{*}$ in which this effect occurs change with the angular scale $\ell$. In other words, whether we can safely extrapolate our results up to

\footnotetext{
${ }^{11}$ In the limit of high frequencies (where Faraday rotation and depolarization vanish), we expect the polarization fraction $p$ to become frequency independent. It may still depend on angular scale $\ell$, however. If it does depend on $\ell$, there is no fundamental reason why it cannot exceed $100 \%$ on some angular scales (even though the polarization at a given point is by definition $\leqslant 100 \%$ )-imagine, say, a uniform synchrotron-emitting plasma with small-scale variations in the magnetic field direction. However, Fig. 7 and Table II show that as the frequency increases, the polarized power spectrum gets progressively redder, providing a tantalizing hint of convergence towards the same power spectrum slope as the total intensity component. If this is actually what happens in the high frequency limit, then the polarization fraction does indeed become a simple constant.
} 


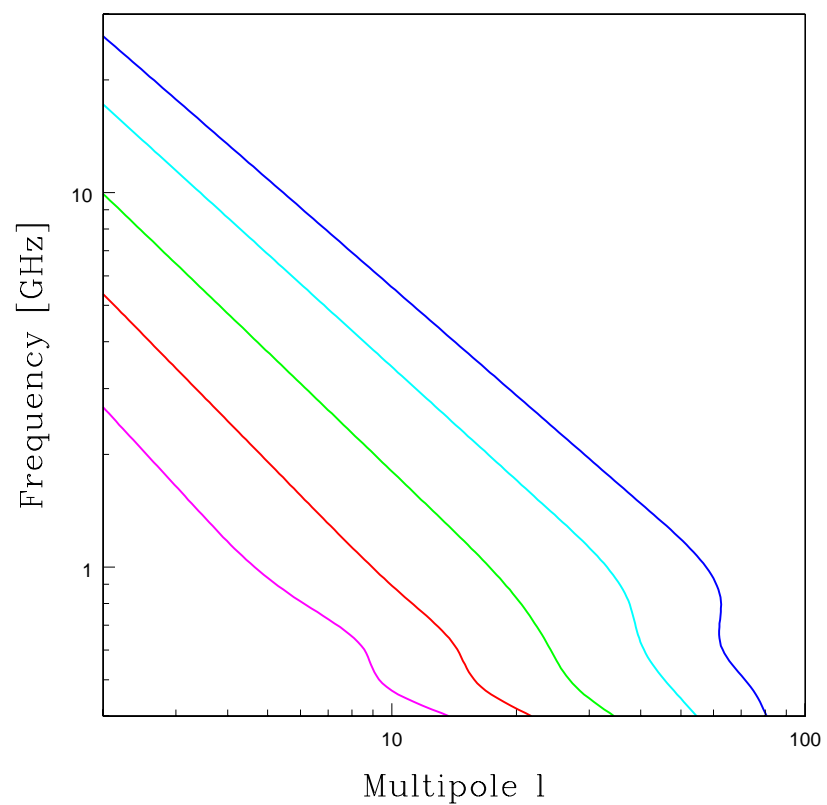

FIG. 10. $(\ell, \nu)$ plane showing contours of constant polarization percentage. From bottom up, the curves are for the $0.01 \%, 0.1 \%$, $1 \%, 10 \%$ and $70 \%$.

CMB frequencies depends not only on the frequency but also in the angular scale. For instance, the contamination of the CMB quadrupole from galactic synchrotron polarization can only be obtained from extrapolations of data at frequencies exceeding $\nu_{*} \sim 10 \mathrm{GHz}$, with $\nu_{*}$ dropping towards smaller angular scales. ${ }^{12}$

All the information above is summarized in Fig. 10, which shows contours of constant polarization percentage $p$ $=\delta T_{\ell}^{E} / \delta T_{\ell}^{T}$ in the two-dimensional $(\ell, \nu)$ plane. In other words, this figure can be interpreted as a contour plot of the depolarization. The depolarization is seen to be negligible at high frequencies and on tiny scales, gradually increasing towards the lower left corner (towards low frequencies and on large angular scales) where Faraday rotation and depolarization effects become important.

This has important implications. For instance, a nice allsky simulation of synchrotron polarization at $\mathrm{CMB}$ frequencies was recently performed assuming that the power spectra of $\cos 2 \theta$ and $\sin 2 \theta$ (where $\theta$ is the polarization angle) were frequency independent [29]. Our results indicate that these two power spectra are dominated by Faraday rotation and depolarization effects, which implies that the $E$ and $B$ power should be mostly due to changes in polarization angle $\theta$, and not to variations in overall intensity - this precise behavior is also seen by [29]. If Faraday rotation and depolarization effects are indeed dominant, then it is not obvious that such

\footnotetext{
${ }^{12}$ Due to the fact that we are dealing with cross-correlations, the results presented here should not be biased by systematic errors or calibration uncertainties in input data, since they would be uncorrelated between the different surveys used. Spurious offsets will not cause excess noise either, since we removed the zero point from each survey before calculating the cross-correlations.
}

frequency extrapolation of the $\cos 2 \theta$ and $\sin 2 \theta$ power spectra are valid.

Figure 9 also shows the POLAR limit of $E<7.4 \mu \mathrm{K}$ from Table I (lower right corner). Since this limit is centered in $\ell \sim 14$, it can be directly compared with the middle curve. The noticeable gap between the two implies that we get interesting constraints from POLAR on foreground models. No synchrotron polarization is detected even though the Haslam stripe shows substantial synchrotron emission in the POLAR region, so either the synchrotron polarization percentage is small or the synchrotron emission falls even more steeply towards higher frequencies than the plotted curves indicate. A spectral index $\alpha=-2.8$ (as shown in the plot) is only allowed if the polarization percentage $p$ is lower than $10 \%$. If $p=20 \%$, then $\alpha<-3.0$, and almost complete polarization (about $70 \%$ is physically possible) would require $\alpha<-3.4$, in poor agreement with theoretical and observational indications [52-54]. In other words, our results suggest a rather low synchrotron polarization percentage at $\mathrm{CMB}$ frequencies. ${ }^{13}$

\section{CONCLUSIONS}

CMB polarization and its decomposition into $E$ and $B$ modes is a topic of growing importance and interest in cosmology. In the era of WMAP, a key issue is to estimate the contribution of galactic foregrounds (more specifically, polarized synchrotron emission) to these modes. We have used the POLAR experiment and radio surveys in order to quantify this contribution at large angular scales.

Using matrix-based quadratic estimator methods, we cross-correlated POLAR with DMR data and obtained upper limits of $E<7.4 \mu \mathrm{K}$ and $|X|<11.1 \mu \mathrm{K}$ at $95 \%$ confidence. These upper limits are, unfortunately, too high to place interesting constrains on reionization models. A similar crosscorrelation analysis was performed by replacing the DMR with the Haslam data, obtaining an upper limit of $|X|$ $\lesssim 15.4 \mu \mathrm{K}$ at $95 \%$ confidence.

We also used our quadratic estimator methods to measure the power spectra from the Leiden surveys, obtaining the following key results:

(1) The synchrotron $E$ and $B$ contributions are equal to within $10 \%$ from 408 to $820 \mathrm{MHz}$, with a hint of $E$ domination at higher frequencies. One interpretation is that $E>B$ at $\mathrm{CMB}$ frequencies but that Faraday rotation mixes the two at low frequencies.

(2) Faraday rotation and depolarization effects depend not only on frequency but also on angular scale-they are important at low frequencies $(\nu \lesssim 10 \mathrm{GHz})$ and on large angular scales.

\footnotetext{
${ }^{13}$ From the COBE/DMR-Haslam cross-correlation results $[87,88]$, we known that the rms galactic signal of the synchrotron emission is lower than $7.1 \mu \mathrm{K}$ at $53 \mathrm{GHz}$. Note that this value is substantially lower than the one we obtain when extrapolating the $\ell \sim 14$ curve of Fig. 9 to the DMR frequencies. This result indicates that $\alpha<-2.8$ or that there is a deviation from the power law behavior at frequencies above a few $\mathrm{GHz}$.
} 
(3) We must take into account Faraday rotation and depolarization effects when extrapolating radio survey results from low to high galactic latitudes and from low to high frequencies.

(4) We detect no significant synchrotron $T E$ cross correlation coefficient $(|r| \leqq 0.2)$, but Faraday rotation we could have hidden a substantial correlation detectable at $\mathrm{CMB}$ frequencies.

(5) Combining the POLAR and radio frequency results, and the fact that the $E$ polarization of the abundant Haslam signal in the POLAR region is not detected at $30 \mathrm{GHz}$, suggests that the synchrotron polarization percentage at CMB frequencies is rather low.
Experiments such as WMAP and Planck will shed significant new light on synchrotron polarization and allow better quantification of its impact both on these experiments and on ground-based CMB observations.

\section{ACKNOWLEDGMENTS}

This work was supported by NSF grants AST-0071213 and AST-0134999 and NASA grants NAG5-9194 and NAG5-11099. M.T. acknowledges the David and Lucile Packard Foundation and the Research Corporation for financial support.
[1] J. Kovac et al., Nature (London) 420, 772 (2002).

[2] M. Zaldarriaga, D.N. Spergel, and U. Seljak, Astrophys. J. 488, 1 (1997).

[3] D.J. Eisenstein, W. Hu, and M. Tegmark, Astrophys. J. 518, 2 (1998).

[4] M. Tegmark, D.J. Eisenstein, W. Hu, and A. de Oliveira-Costa, Astrophys. J. 530, 133 (2000).

[5] M. Kaplinghat et al., Astrophys. J. 583, 24 (2003).

[6] A.A. Penzias and R.W. Wilson, Astrophys. J. 142, 419 (1965).

[7] N. Caderni, Phys. Rev. D 17, 1908 (1978).

[8] G. Nanos, Astrophys. J. 232, 341 (1979).

[9] P.M. Lubin and G.F. Smoot, Phys. Rev. Lett. 42, 129 (1979).

[10] P.M. Lubin and G.F. Smoot, Astrophys. J. 245, 1 (1981).

[11] G. Sironi, G. Boella, G. Bonelli, L. Brunetti, F. Cavaliere, M. Fervasi, G. Giardino, and A. Passerini, New Astron. 3, 1 (1998).

[12] P.M. Lubin, P. Melese, and G.F. Smoot, Astrophys. J. Lett. 273, L51 (1983).

[13] E.J. Wollack, N.C. Jarosik, C.B. Netterfield, L.A. Page, and D. Wilkinson, Astrophys. J. Lett. 419, L49 (1993).

[14] C.B. Netterfield et al., Astrophys. J. 474, 47 (1997).

[15] E. Torbet, M.J. Devlin, W.B. Dorwart, T. Herbig, A.D. Miller, M.R. Nolta, L.A. Page, J. Puchalla, and H.T. Tran, Astrophys. J. Lett. 521, L79 (1999).

[16] R.B. Partridge, J. Nawakowski, and H.M. Martin, Nature (London) 311, 146 (1988).

[17] E.B. Fomalont, R.B. Partridge, J.D. Lowenthal, and R.A. Windhorst, Astrophys. J. 404, 8 (1993).

[18] R.B. Partridge, E.A. Richards, E.B. Fomalont, K.I. Kellerman, and R.A. Windhorst, Astrophys. J. 483, 38 (1997).

[19] R. Subrahmanyan, M.J. Kesteven, R.D. Ekers, M. Sinclair, and J. Silk, Mon. Not. R. Astron. Soc. 315, 808 (2000).

[20] G.F. Smoot, astro-ph/9902027.

[21] M. Hedman et al., astro-ph/0204438.

[22] B. Keating et al., Astrophys. J. 560, 1 (2001).

[23] A. de Oliveira-Costa et al., Phys. Rev. D 67, 023003 (2003).

[24] M. Tucci, E. Carretti, S. Cecchini, R. Fabbri, M. Orsini, and E. Pierpaoli, astro-ph/0006387.

[25] C. Baccigalupi, C. Burigana, F. Perrotta, G. De Zotti, L. La Porta, D. Maino, M. Maris, and R. Paladini, astro-ph/0009135.

[26] C. Burigana and L. La Porta, astro-ph/0202439.

[27] M. Bruscoli, M. Tucci, V. Natale, E. Carretti, R. Fabbri, C.
Sbarra, and S. Cortiglioni, astro-ph/0202389.

[28] M. Tucci, E. Carretti, S. Cecchini, L. Nicastro, R. Fabbri, B.M. Gaensler, J.M. Dickey, and N.M. McClure-Griffiths, Astrophys. J. 579, 607 (2002).

[29] G. Giardino, A.J. Banday, K.M. Gorski, K. Bennett, J.L. Jonas, and J. Tauber, Astron. Astrophys. 387, 82 (2002).

[30] A. Kamionkowski, A. Kosowsky, and A. Stebbins, Phys. Rev. D 55, 7368 (1997).

[31] M. Zaldarriaga and U. Seljak, Phys. Rev. D 55, 1830 (1997).

[32] M. Zaldarriaga, Astrophys. J. 503, 1 (1998).

[33] W. Hu and M. White, Phys. Rev. D 56, 596 (1997).

[34] G. Westerhout, C.L. Seeger, W.N. Brouw, and J. Tinbergen, Bull. Astron. Inst. Neth. 16, 187 (1962).

[35] T.A.T. Spoelstra, Astron. Astrophys. 135, 238 (1984).

[36] W.N. Brouw and T.A.T. Spoelstra, Astron. Astrophys., Suppl. Ser. 26, 129 (1976).

[37] N. Junkes, E. Fuerst, and W. Reich, Astron. Astrophys., Suppl. Ser. 69, 451 (1987).

[38] M.H. Wieringa, A.G. De Bruyn, D. Jansen, W.N. Brouw, and P. Katgert, Astron. Astrophys. 268, 215 (1993).

[39] A.R. Duncan, R.T. Stewart, R.F. Haynes, and K.L. Jones, Mon. Not. R. Astron. Soc. 277, 36 (1995).

[40] A.R. Duncan, R.F. Haynes, K.L. Jones, and R.T. Stewart, Mon. Not. R. Astron. Soc. 291, 279 (1997).

[41] B. Uyaniker, E. Fuerst, W. Reich, P. Reich, and R. Wielebinski, astro-ph/9807013.

[42] B. Uyaniker, E. Fuerst, W. Reich, P. Reich, and R. Wielebinski, astro-ph/9905023.

[43] M. Zaldarriaga, Phys. Rev. D 55, 1822 (1997).

[44] M. Kamionkowski and A. Kosowsky, Annu. Rev. Nucl. Part. Sci. 49, 77 (1999).

[45] X. Chen and M. Kamionkowski, Phys. Rev. D 60, 104036 (1999).

[46] M. Kamionkowski and A.H. Jaffe, Int. J. Mod. Phys. A 16, 116 (2001).

[47] M. Zaldarriaga and U. Seljak, Phys. Rev. D 58, 023003 (1998).

[48] G. B. Rybicki and A. P. Lightman, Radiative Processes in Astrophysics (John Wiley and Sons, New York, 1979), pp. 167194.

[49] R.D. Davies and A. Wilkinson, astro-ph/9804208.

[50] G.F. Smoot, in Microwave Foregrounds, ASP Conference Se- 
ries, edited by A. de Oliveira-Costa and M. Tegmark (ASP, San Francisco, 1999), p. 61.

[51] P. Platania et al., Astrophys. J. 505, 473 (1998).

[52] A.J. Banday and A.W. Wolfendale, Mon. Not. R. Astron. Soc. 248, 705 (1991).

[53] J.L. Jonas, Ph.D. thesis, Rhodes University, South Africa, 1999.

[54] R.S. Roger, C.H. Costain, T.L. Landecker, and C.M. Swerdlyk, astro-ph/9902213.

[55] C.G.T. Haslam et al., Astron. Astrophys. 100, 209 (1981).

[56] C.G.T. Haslam et al., Astron. Astrophys., Suppl. Ser. 47, 1 (1982).

[57] D. Finkbeiner et al. (in preparation).

[58] M. Tegmark and G. Efstathiou, Mon. Not. R. Astron. Soc. 281, 1297 (1996).

[59] F.R. Bouchet, R. Gispert, and J.L. Puget, in Unveiling the CIB, edited by E. Dwek, AIP Conf. Proc. No. 348 (AIP, Woodbury, NY, 1996), p. 225.

[60] F.R. Bouchet and R. Gispert, New Astron. 4, 443 (1999).

[61] A. de Oliveira-Costa et al., Astrophys. J. 530, 133 (2002).

[62] G. Giardino, A.J. Banday, P. Fosalba, K.M. Gorski, J.L. Jonas, W. O'Mullane, and J. Tauber, astro-ph/0103233.

[63] W. Reich, E. Fuerst, P. Reich, R. Wielebinski, and M. Wolleben, astro-ph/0111437.

[64] A.R. Duncan, P. Reich, W. Reich, and E. Fuerst, Astron. Astrophys. 350, 447 (1999).

[65] P. Reich and W. Reich, Astron. Astrophys., Suppl. Ser. 74, 7 (1998)

[66] A.R. Duncan, in Galactic Foreground Polarization, edited by E.M. Berkhuijsen (MPIfR, Bonn, 1999), p. 58.

[67] D.D. Sokoloff et al., Mon. Not. R. Astron. Soc. 299, 189 (1998).
[68] K. Rohlfs and T. L. Wilson, Tools of Radio Astronomy, 1996, pp. $43-47$.

[69] M. Haverkorn, P. Katgert, and A.G. de Bruyn, astro-ph/0003260.

[70] B.M. Gaensler, J.M. Dickey, N.M. McClure-Griffiths, N.S. Bizunok, and A.J. Green, astro-ph/0010518.

[71] S. Cortiglioni and T.A.T. Spoelstra, Astron. Astrophys. 302, 1 (1995).

[72] T.A.T. Spoelstra, Astron. Astrophys. 13, 237 (1971).

[73] T.A.T. Spoelstra, Astron. Astrophys. 21, 61 (1972).

[74] J.R. Bond, A.H. Jaffe, and L.E. Knox, Astrophys. J. 533, 19 (2000).

[75] M. Tegmark and A. de Oliveira-Costa, Phys. Rev. D 64, 063001 (2001).

[76] A.H. Jaffe, M. Kamionkowski, and L. Wang, Phys. Rev. D 61, 083501 (2000).

[77] M. Zaldarriaga, Phys. Rev. D 64, 103001 (2001).

[78] A. Lewis, A. Challinor, and N. Turok, Phys. Rev. D 65, 023505 (2002).

[79] E.F. Bunn, Phys. Rev. D 65, 043003 (2002).

[80] E.F. Bunn, M. Zaldarriaga, M. Tegmark, and A. de OliveiraCosta, Phys. Rev. D 67, 023501 (2003).

[81] B. Keating et al., Astrophys. J., Suppl. Ser. 144, 1 (2002).

[82] C. O’Dell et al., Phys. Rev. D 68, 042002 (2003).

[83] G.F. Smoot et al., Astrophys. J. Lett. 396, L1 (1992).

[84] U. Seljak and M. Zaldarriaga, Astrophys. J. 469, 437 (1996).

[85] X. Wang, M. Tegmark, and M. Zaldarriaga, Phys. Rev. D 65, 123001 (2002).

[86] G. Efstathiou et al., astro-ph/0109152.

[87] A. Kogut et al., Astrophys. J. 460, 1 (1996).

[88] A. Kogut et al., Astrophys. J. Lett. 464, L5 (1996). 